\title{
LONG-DISTANCE HIGH-FIDELITY TELEPORTATION USING SINGLET STATES
}

\author{
Jeffrey H. Shapiro \\ Department of Electrical Engineering and Computer Science \\ Research Laboratory of Electronics \\ Laboratory for Information and Decision Systems \\ Massachusetts Institute of Technology \\ Cambridge, MA 02139
}

\begin{abstract}
A quantum communication system is proposed that uses polarization-entangled photons and trapped-atom quantum memories. This system is capable of long-distance, highfidelity teleportation, and long-duration quantum storage.
\end{abstract}

\section{INTRODUCTION}

The preeminent obstacle to the development of quantum information technology is the difficulty of transmitting quantum information over noisy and lossy quantum communication channels, recovering and refreshing the quantum information that is received, and then storing it in a reliable quantum memory. This paper proposes a singlet-based approach to quantum communication that uses a novel ultrabright narrowband source of polarization-entangled photon pairs, ${ }^{1}$ and a trapped-atom quantum memory ${ }^{2}$ whose loading can be nondestructively verified and whose structure permits all four Bell-state measurements to be performed. The system is designed to operate with standard telecommunication fiber as its transmission medium. It can achieve a loss-limited throughput as high as 200 entangled-pairs/sec with a $97.5 \%$ fidelity over a $50 \mathrm{~km}$ path when there is $10 \mathrm{~dB}$ of fixed loss in the overall system and $0.2 \mathrm{~dB} / \mathrm{km}$ propagation loss in the fiber. This long-distance high-fidelity quantum transmission is accomplished without the use of entanglement swapping, i.e., no quantum repeaters, and without the use of entanglement purification or quantum error correction. 


\section{TELEPORTATION USING SINGLET STATES}

The notion that singlet states could be used to achieve teleportation is due to Bennett et al. ${ }^{3}$ The transmitter and receiver stations share the entangled qubits of a singlet state, $|\psi\rangle_{\mathrm{TR}}=\left(|0\rangle_{\mathrm{T}}|1\rangle_{\mathrm{R}}-|1\rangle_{\mathrm{T}}|0\rangle_{\mathrm{R}}\right) / \sqrt{2}$. The transmitter accepts an input-mode qubit, $|\Psi\rangle_{\text {in }}=\alpha|0\rangle_{\text {in }}+\beta|1\rangle_{\text {in }}$, leaving the input-mode, transmitter, and receiver in the joint state $|\Psi\rangle_{\text {in }}|\psi\rangle_{\mathrm{TR}}$. Making the Bell-state measurements, $\left\{\left(|1\rangle_{\text {in }}|0\rangle_{\mathrm{T}} \pm|0\rangle_{\text {in }}|1\rangle_{\mathrm{T}}\right) / 2\right.$, $\left.\left(|1\rangle_{\text {in }}|1\rangle_{\mathrm{T}} \pm|0\rangle_{\text {in }}|0\rangle_{\mathrm{T}}\right) / 2\right\}$, on the joint input-mode/transmitter system then yields the two bits of classical information that the receiver needs to reconstruct the input state, i.e., to complete the teleportation process. An initial experimental demonstration of teleportation using singlet states was performed by the Innsbruck group., ${ }^{4,5}$ There were several significant limitations to this initial demonstration, however, which preclude its forming the basis for useful long-distance quantum-state communication. First, because only one of the Bell states was measured, the demonstration was conditional: teleportation only occurred when the input-mode/transmitter state projected onto that Bell state. Second, the demonstration was a table-top experiment: there was no provision for long-distance transmission. Finally, the demonstration did not include a quantum memory: the teleported state could not be stored for application to quantum cryptography or quantum computation. In the next section we outline our proposal for a singlet-based quantum communication system that remedies all of these limitations.

\section{LONG-DISTANCE TELEPORTATION WITH QUANTUM MEMORY}

Consider the quantum communication system shown in Fig. 1. An ultrabright nar-

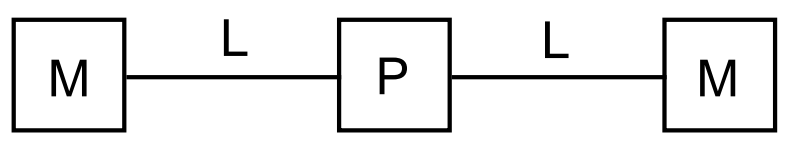

Figure 1. Schematic of long-distance quantum communication system: $P=$ ultrabright narrowband source of polarization-entangled photon pairs; $L=L \mathrm{~km}$ of standard telecommunication fiber; $M=$ trapped-atom quantum memory.

rowband source of polarization-entangled photon pairs ${ }^{1}$ launches the entangled qubits from a singlet state into two $L$-km-long standard telecommunication fibers. The photons emerging from the fibers are then loaded into trapped-atom quantum memories. ${ }^{2}$ These memories store the photon-polarization qubits in long-lived hyperfine levels. Because it is compatible with fiber-optic transmission, this configuration is capable of long-distance teleportation. Because of the long decoherence times that can be realized with trapped atoms, this configuration supports long-duration quantum storage. We devote the rest of this section to summarizing the basic features of our proposal.

Each $M$ block in Fig. 1 is a quantum memory in which a single ultra-cold ${ }^{87} \mathrm{Rb}$ atom ( $\sim 6 \mathrm{MHz}$ linewidth) is confined by a $\mathrm{CO}_{2}$-laser trap in an ultra-high vacuum chamber with cryogenic walls within a high-finesse $(\sim 15 \mathrm{MHz}$ linewidth) single-ended optical cavity. This memory can absorb a $795 \mathrm{~nm}$ photon, in an arbitrary polarization state, transferring the qubit from the photon to the degenerate $B$ levels of Fig. 2a and thence to long-lived storage levels, by coherently driving the $B$-to- $D$ transitions. (We are using abstract symbols here for the hyperfine levels of rubidium, see Ref. 2 for the actual atomic levels involved as well as a complete description of the memory and its operation.) With a liquid helium cryostat, so that the background pressure is less than $10^{-14}$ Torr, the expected lifetime of the trapped rubidium atom will be more than an 
hour. Moreover, the decoherence time can be expected to be about the same as this lifetime for the levels we have chosen to use for storage. By using optically off-resonant Raman (OOR) transitions, the Bell states of two atoms in a single vacuum-chamber trap can be converted to superposition states of one of the atoms. All four Bell measurements can then be made, sequentially, by detecting the presence (or absence) of fluorescence as an appropriate sequence of OOR laser pulses is applied to the latter atom. The Bellmeasurement results (two bits of classical information) in one memory can be sent to a distant memory, where (at most) two additional OOR pulses are needed to complete the Bennett et al. state transformation. The qubit stored in a trapped rubidium atom can be converted back into a photon - with the same polarization information as the one whose qubit was stored-by reversing the Raman excitation process that occurs during memory loading.
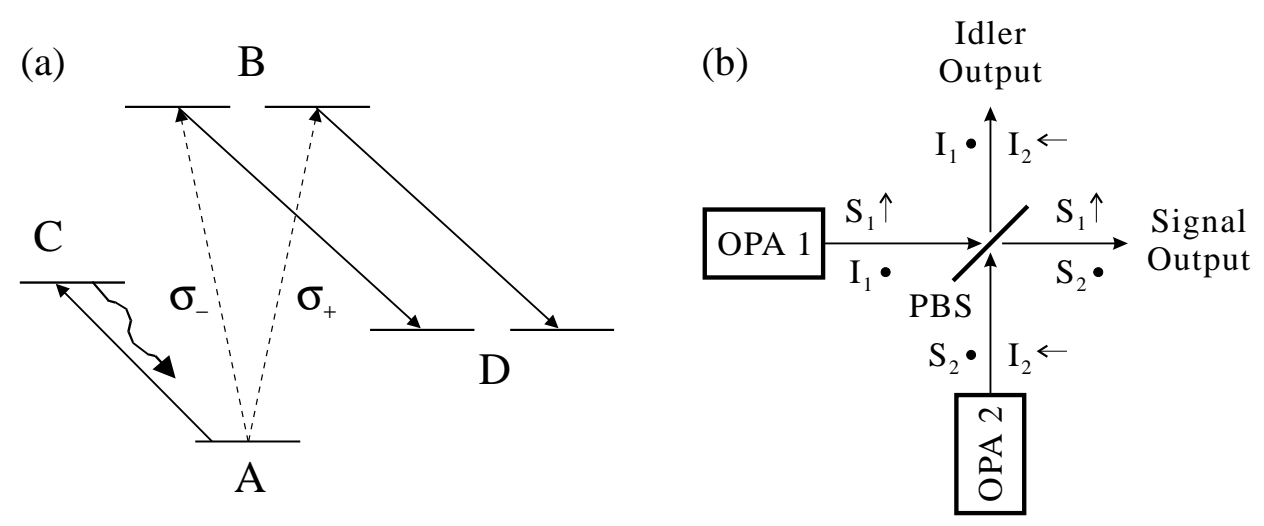

Figure 2. Essential components of singlet-state quantum communication system from Fig. 1. Left (a), simplified atomic-level schematic of the trapped rubidium atom quantum memory: $A$-to- $B$ transition occurs when one photon from an entangled pair is absorbed; $B$-to- $D$ transition is coherently driven to enable storage in the long-lived $D$ levels; $A$-to- $C$ cycling transition permits nondestructive determination of when a photon has been absorbed. Right (b), ultrabright narrowband source of polarization-entangled photon pairs: each optical parametric amplifier (OPA1 and OPA2) is type-II phase matched; for each optical beam the propagation direction is $\hat{z}$, and $\hat{x}$ and $\hat{y}$ polarizations are denoted by arrows and bullets, respectively; PBS, polarizing beam splitter.

The $P$-block in Fig. 1 is an ultrabright narrowband source of polarization-entangled photon pairs, capable of producing $\sim 10^{6}$ pairs $/$ sec in $\sim 30 \mathrm{MHz}$ bandwidth by appropriately combining the signal and idler output beams from two doubly-resonant type-II phase-matched optical parametric amplifiers (OPAs), as sketched in Fig. 2b. ${ }^{1}$ The Innsbruck teleportation experiment used parametric downconversion as its source of polarization-entangled photon pairs. This process is intrinsically very broadband ( 10 $0^{13} \mathrm{~Hz}$ bandwidth), whereas a trapped-atom quantum memory only absorbs photons within a very narrow $\left(\sim 10^{7} \mathrm{~Hz}\right)$ bandwidth. As a result, the brightest downconverter source reported to date ${ }^{6}$ might only produce $\sim 15$ pairs/sec in this narrow optical bandwidth. This is why an ultrabright narrowband source is so essential. Our source has the following properties. The fluorescence spectrum of the signal and idler beams is controlled by the doubly-resonant OPA cavities. These can be advantageously and easily tailored to produce the desired (factor-of-two broader than the memory-cavity's) bandwidth. By using periodically-poled potassium titanyl phosphate (PPKTP), a quasi-phase-matched type-II nonlinear material, we can produce $\sim 10^{6}$ pairs $/ \mathrm{sec}$ at the $795 \mathrm{~nm}$ wavelength of the rubidium memory for direct memory-loading (i.e., localstorage) applications. For long-distance transmission to remotely-located memories, we use a different PPKTP crystal and pump wavelength to generate $\sim 10^{6}$ pairs $/ \mathrm{sec}$ in 
the 1.55- $\mu \mathrm{m}$-wavelength low-loss fiber transmission window. After fiber propagation we then shift the entanglement to the $795 \mathrm{~nm}$ wavelength needed for the rubidium-atom memory via quantum-state frequency translation, a procedure previously proposed and demonstrated by Kumar ${ }^{7,8}$ and shown schematically in Fig. 3.

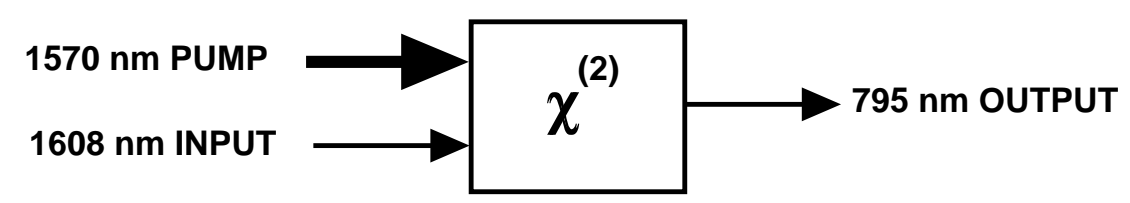

Figure 3. Schematic of quantum frequency conversion: a strong pump beam at 1570 nm converts a qubit photon received at $1608 \mathrm{~nm}$ (in the low-loss fiber transmission window) to a qubit photon at the $795 \mathrm{~nm}$ wavelength of the ${ }^{87} \mathrm{Rb}$ quantum memory.

Transmission in the $1.55-\mu \mathrm{m}$-wavelength window is not enough to make singletbased quantum communication fully compatible with standard telecommunication fiber. It is also crucial to ensure that polarization is not degraded by the propagation process. Our scheme for polarization maintenance, shown schematically in Fig. 4, relies on time-division multiplexing. Time slices from the signal beams from our two OPAs are sent down one fiber in the same linear polarization but in nonoverlapping time slots, accompanied by a strong out-of-band laser pulse. By tracking and restoring the linear polarization of the strong pulse, we can restore the linear polarization of the signalbeam time slices at the far end of the fiber. After this linear-polarization restoration, we then reassemble a time-epoch of the full vector signal beam by delaying the first time slot and combining it on a polarizing beam splitter (PBS) with the second time slot after the latter has had its linear polarization rotated by $90^{\circ}$. A similar procedure is performed to reassemble idler time-slices after they have propagated down the other fiber in Fig. 1. In effect, this replaces the source-located passive PBS in Fig. 2b with a time-gated memory-located polarization combiner at the far end of each fiber. This approach, which is inspired by the Bergman et al. two-pulse fiber-squeezing experiment, ${ }^{9}$ common-modes out the vast majority of the phase fluctuations and the polarization birefringence incurred in the fiber, permitting standard telecommunication fiber to be used in lieu of the lossier and much more expensive polarization-maintaining fiber.

\section{LOSS-LIMITED PERFORMANCE ANALYSIS}

Quantum communication is carried out in the Fig. 1 configuration via the following protocol. The entire system is clocked. Time slots of signal and idler (say $400 \mathrm{~ns}$ long) are transmitted down optical fibers to the quantum memories. These slots are gated into the memory cavities - with their respective atoms either physically displaced or optically detuned so that no $A$-to- $B$ (i.e., no $795 \mathrm{~nm}$ ) absorptions occur. After a short loading interval (a few cold-cavity lifetimes, say $400 \mathrm{~ns}$ ), each atom is moved (or tuned) into the absorbing position and $B$-to- $D$ coherent pumping is initiated. After about $100 \mathrm{~ns}$, coherent pumping ceases and the $A$-to- $C$ cycling transition (shown in Fig. 2a) is repeatedly driven (say 30 times, taking nearly $1 \mu \mathrm{s}$ ). (To avoid spontaneous decay from $B$, the two-step storage sequence can be compressed into a single off-resonant Raman transition. ${ }^{10,11}$ ) By monitoring a cavity for the fluorescence from this cycling transition, we can reliably detect whether or not a $795 \mathrm{~nm}$ photon has been absorbed by the atom in that cavity. If neither atom or if only one atom has absorbed such a photon, then we cycle both atoms back to their $A$ states and start anew. If no cycling-transition fluorescence is detected in either cavity, then, because we have employed enough cycles 


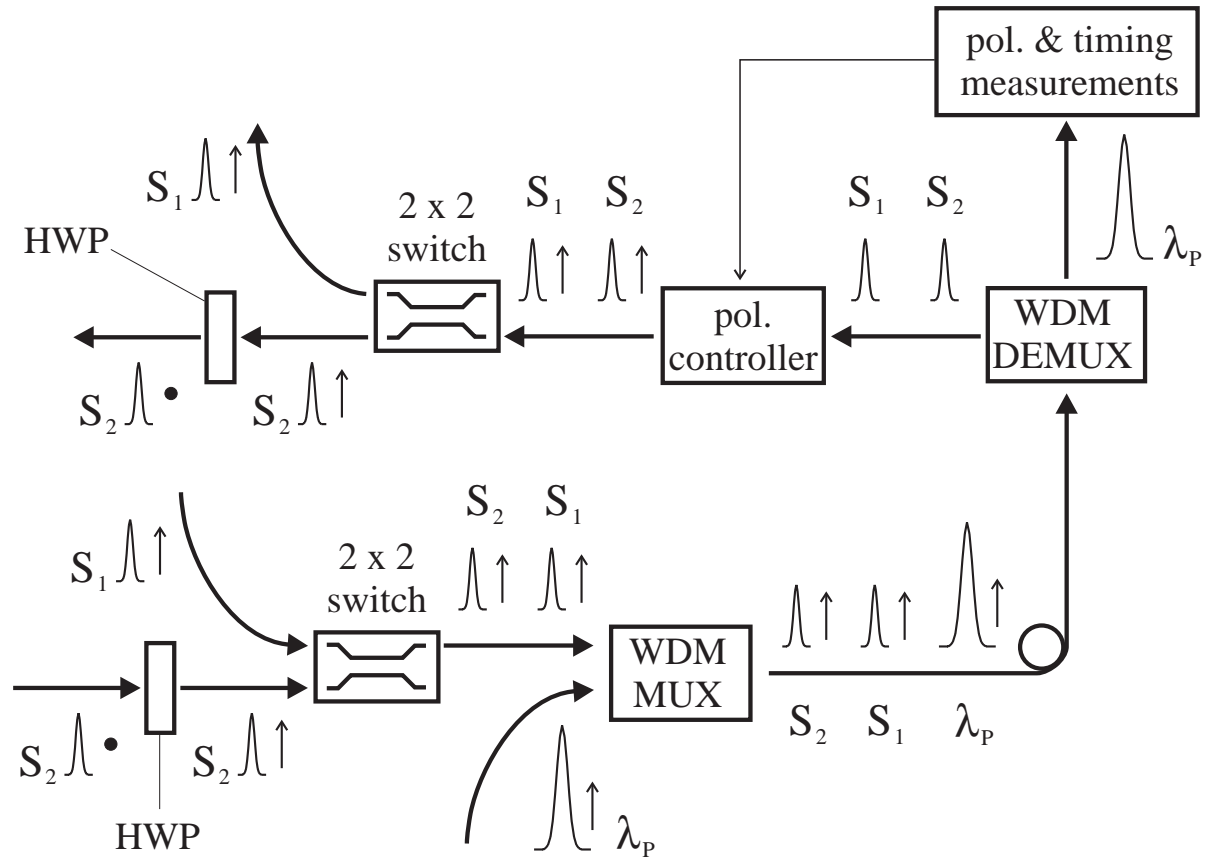

Figure 4. Transmission of time-division multiplexed signal beams from OPA1 and OPA2 through an optical fiber. With the use of a half-wave plate (HWP) the signal pulses and the pilot pulse are linearly polarized the same way. The pilot pulse - at a wavelength $\lambda_{p}$ which is different from the signal or idler wavelengths - is injected into and extracted from the fiber using a wavelength-division multiplexer (WDM MUX) and a wavelength-division demultiplexer (WDM DEMUX), respectively. Not shown is the final polarizing beam splitter for combining the two signal-beam outputs, cf. Fig. 2b. A similar overall arrangement is used to transmit the idler beams from OPA1 and OPA2.

to ensure very high probability of detecting that the atom is in its $A$ state, it must be that both atoms have absorbed $795 \mathrm{~nm}$ photons and stored the respective qubit information in their long-lived degenerate $D$ levels. These levels are not resonant with the laser driving the cycling transition, and so the loading of our quantum memory is nondestructively verified in this manner.

We expect that the preceding memory-loading protocol can be run at rates as high as $R=500 \mathrm{kHz}$, i.e., we can get an independent try at loading an entangled photon pair into the two memory elements of Fig. 1 every $2 \mu \mathrm{s}$. With a high probability, $P_{\text {erasure }}$, any particular memory-loading trial will result in an erasure, i.e., propagation loss and other inefficiencies combine to preclude both atoms from absorbing photons in the same time epoch. With a small probability, $P_{\text {success }}$, the two atoms will absorb the photons from a single polarization-entangled pair, viz., we have a memory-loading success. With a much smaller probability, $P_{\text {error }}$, both atoms will have absorbed photons but these photons will not have come from a single polarization-entangled pair; this is the error event. These three events constitute a complete taxonomy of loading-trial possibilities, i.e., their respective probabilities sum to unity.

In terms of $\left\{R, P_{\text {erasure }}, P_{\text {success }}, P_{\text {error }}\right\}$ it is easy to identify the key figures-of-merit for the Fig. 1 configuration. First, there is its robustness to propagation losses and other inefficiencies. These effects merely increase $P_{\text {erasure }}$ and hence reduce the throughput, i.e., the number of successful entanglement-loadings/sec, $N_{\text {success }} \equiv R P_{\text {success }}$, that could be achieved if the quantum memories each contained a lattice of trapped atoms for sequential loading of many pairs. It is the loading errors, which occur with probability $P_{\text {error }}$, that provide the ultimate limit on the entanglement fidelity of the Fig. 1 configuration. This loss-limited fidelity is given by $F_{\max }=1-P_{\text {error }} / 2\left(P_{\text {success }}+P_{\text {error }}\right)$, where 
we have assumed that the error event loads independent, randomly-polarized photons into each memory.

\section{OPA Statistics}

To quantify the loss-limited throughput and entanglement fidelity of the Fig. 1 system we must first quantify the behavior of the dual-OPA source. We begin by showing that this source - under ideal lossless conditions - does indeed produce the desired singlet state. Assume matched signal and idler cavities, each with linewidth $\Gamma$, zero detuning, and no excess loss. Also assume anti-phased pumping at a fraction, $G^{2}$, of oscillation threshold, with no pump depletion or excess noise. From Ref. 1 we then have that the output beams from OPAs 1 and 2 are in an entangled, zero-mean Gaussian pure state, which is completely characterized by the following normally-ordered and phase-sensitive correlation functions:

$$
\begin{aligned}
& \left\langle\hat{A}_{k_{j}}^{\dagger}(t+\tau) \hat{A}_{k_{j}}(t)\right\rangle=\frac{G \Gamma}{2}\left[\frac{\exp [-(1-G) \Gamma|\tau|]}{1-G}-\frac{\exp [-(1+G) \Gamma|\tau|]}{1+G}\right], \\
& \left\langle\hat{A}_{S_{j}}(t+\tau) \hat{A}_{I_{j}}(t)\right\rangle=\frac{(-1)^{j-1} G \Gamma}{2}\left[\frac{\exp [-(1-G) \Gamma|\tau|]}{1-G}+\frac{\exp [-(1+G) \Gamma|\tau|]}{1+G}\right],
\end{aligned}
$$

where $\left\{\hat{A}_{k_{j}}(t) e^{-i \omega_{k} t}: k=S\right.$ (signal), $I$ (idler), $\left.j=1,2\right\}$ are positive-frequency, photonunits OPA-output field operators.

After combining the outputs of OPAs 1 and 2 into vector fields $\hat{\vec{A}}_{S}(t)$ and $\hat{\vec{A}}_{I}(t)$, we can show that the Fourier component of the vector-signal field at frequency $\omega_{S}+\Delta \omega$ and the vector-idler Fourier component at frequency $\omega_{I}-\Delta \omega$ are in the entangled Bose-Einstein state,

$$
|\psi\rangle_{\mathrm{SI}}=\sum_{n=0}^{\infty} \sqrt{\frac{\bar{N}^{n}}{(\bar{N}+1)^{n+1}}}|n\rangle_{S_{x}}|n\rangle_{I_{y}} \sum_{n=0}^{\infty}(-1)^{n} \sqrt{\frac{\bar{N}^{n}}{(\bar{N}+1)^{n+1}}}|n\rangle_{S_{y}}|n\rangle_{I_{x}}
$$

in number-ket representation, where $\bar{N}=4 G^{2} /\left[\left(1-G^{2}-\Delta \omega^{2} / \Gamma^{2}\right)^{2}+4 \Delta \omega^{2} / \Gamma^{2}\right]$ is the average photon number per mode at detuning $\Delta \omega$. For $\bar{N} \ll 1$, this joint state reduces to,

$|\psi\rangle_{\mathrm{SI}} \approx \frac{1}{\bar{N}+1}|0\rangle_{S_{x}}|0\rangle_{I_{y}}|0\rangle_{S_{y}}|0\rangle_{I_{x}}+\sqrt{\frac{\bar{N}}{(\bar{N}+1)^{3}}}\left(|1\rangle_{S_{x}}|1\rangle_{I_{y}}|0\rangle_{S_{y}}|0\rangle_{I_{x}}-|0\rangle_{S_{x}}|0\rangle_{I_{y}}|1\rangle_{S_{y}}|1\rangle_{I_{x}}\right)$

i.e., it is predominantly vacuum, augmented by a small amount of the singlet state.

The presence of excess loss within the OPA cavities, and/or propagation loss along the fiber can be incorporated into this OPA analysis in a straightforward manner. ${ }^{12}$ Assuming symmetric operation, in which the signal and idler encounter identical intracavity and fiber losses, then the correlation-function formulas, Eqs. 11 and 2, are merely multiplied by $\eta_{L} \gamma / \Gamma$, where $\eta_{L}<1$ is the transmission through the fiber $\left(\eta_{L}=10^{0.02 L}\right.$ for $L \mathrm{~km}$ of $0.2 \mathrm{~dB} / \mathrm{km}$-loss fiber), and $\gamma<\Gamma$ is the output-coupling rate of the OPA cavity.

\section{Cavity-Loading Statistics}

To analyze our cold-cavity loading protocol, we relate the annihilation operators of the internal cavity modes - over the $T_{c}$-sec-long loading interval - to the incoming 
signal and idler field operators as follows:

$$
\hat{\vec{a}}_{k}\left(T_{c}\right)=\hat{\vec{a}}_{k}(0) e^{-\Gamma_{c} T_{c}}+\int_{0}^{T_{c}} d t e^{-\Gamma_{c}\left(T_{c}-t\right)}\left[\sqrt{2 \gamma_{c}} \hat{\vec{A}}_{k}(t)+\sqrt{2\left(\Gamma_{c}-\gamma_{c}\right)} \hat{\vec{A}}_{k_{v}}(t)\right],
$$

for $k=S, I$, where $\gamma_{c}<\Gamma_{c}$ is the input-coupling rate and $\Gamma_{c}$ is the linewidth of the (assumed to be identical for signal and idler) memory cavities. The initial intracavity operators and the loss-operators, $\left\{\hat{\vec{a}}_{k}(0), \hat{\vec{A}}_{k_{v}}(t)\right\}$, are in vacuum states.

It is now easy to show that the joint density operator (state) for $\left\{\hat{\vec{a}}_{S}\left(T_{c}\right), \hat{\vec{a}}_{I}\left(T_{c}\right)\right\}$, takes the factored form, $\hat{\rho}_{\vec{S} \vec{I}}=\hat{\rho}_{S_{x} I_{y}} \hat{\rho}_{S_{y} I_{x}}$, where the two-mode density operators on the right-hand side are Gaussian mixed states given by the anti-normally ordered characteristic functions,

$$
\begin{aligned}
\operatorname{tr}\left[\hat{\rho}_{S_{x} I_{y}} e^{-\zeta_{S}^{*} \hat{a}_{S_{x}}-\zeta_{I}^{*} \hat{a}_{I y}} e^{\zeta_{S} \hat{a}_{S x}^{\dagger}+\zeta_{I} \hat{a}_{I y}^{\dagger}}\right] & =\operatorname{tr}\left[\hat{\rho}_{S_{y} I_{x}} e^{-\zeta_{S}^{*} \hat{a}_{S y}+\zeta_{I}^{*} \hat{a}_{I_{x}}} e^{\zeta_{S} \hat{a}_{S_{y}}^{\dagger}-\zeta_{I} \hat{a}_{I_{x}}^{\dagger}}\right] \\
& =\exp \left[-(1+\bar{n})\left(\left|\zeta_{S}\right|^{2}+\left|\zeta_{I}\right|^{2}\right)+2 \tilde{n} \operatorname{Re}\left(\zeta_{S} \zeta_{I}\right)\right],
\end{aligned}
$$

where $\bar{n} \equiv I_{-}-I_{+}$and $\tilde{n} \equiv I_{-}+I_{+}$, with $I_{\mp} \equiv \eta_{L} \gamma \gamma_{c} / \Gamma_{c}(1 \mp G)\left[(1 \mp G) \Gamma+\Gamma_{c}\right]$.

\section{Throughput and Fidelity Calculations}

To calculate the throughput and fidelity of our singlet-based quantum communication system, we need only use the loaded-cavity state, presented in the previous subsection, to find the erasure, success, and error probabilities via,

$$
\begin{aligned}
P_{\text {erasure }} & =\left({ }_{S_{x}}\left\langle 0\left|\hat{\rho}_{S_{x}}\right| 0\right\rangle_{S_{x}}\right)\left({ }_{S_{y}}\left\langle 0\left|\hat{\rho}_{S_{y}}\right| 0\right\rangle_{S_{y}}\right)+\left(_{I_{x}}\left\langle 0\left|\hat{\rho}_{I_{x}}\right| 0\right\rangle_{I x}\right)\left({ }_{I_{y}}\left\langle 0\left|\hat{\rho}_{I_{y}}\right| 0\right\rangle_{I_{y}}\right) \\
& -\left({ }_{S_{x}}\left\langle\left. 0\right|_{I_{y}}\left\langle 0\left|\hat{\rho}_{S_{x} I_{y}}\right| 0\right\rangle_{I_{y}} \mid 0\right\rangle_{S_{x}}\right)\left({ }_{S_{y}}\left\langle\left. 0\right|_{I_{x}}\left\langle 0\left|\hat{\rho}_{S_{y} I_{x}}\right| 0\right\rangle_{I_{x}} \mid 0\right\rangle_{S_{y}}\right), \\
P_{\text {success }} & ={ }_{\mathrm{SI}}\left\langle\psi\left|\hat{\rho}_{\vec{S} \vec{I}}\right| \psi\right\rangle_{\mathrm{SI}} \quad \text { and } \quad P_{\text {error }}=1-P_{\text {erase }}-P_{\text {success }},
\end{aligned}
$$

where $|\psi\rangle_{\mathrm{SI}} \equiv\left(|1\rangle_{S_{x}}|1\rangle_{I_{y}}|0\rangle_{S_{y}}|0\rangle_{I_{x}}-|0\rangle_{S_{x}}|0\rangle_{I_{y}}|1\rangle_{S_{y}}|1\rangle_{I_{x}}\right) / \sqrt{2}$, is the singlet state.

In Fig. 5 we have plotted the throughput and loss-limited fidelity for our quantum communication system under the following assumptions: OPAs pumped at $1 \%$ of their oscillation thresholds $\left(G^{2}=0.01\right) ; 5 \mathrm{~dB}$ of excess loss in each $P$-to- $M$ block path in Fig. $1 ; 0.2 \mathrm{~dB} / \mathrm{km}$ loss in each fiber; $\Gamma_{c} / \Gamma=0.5$; and $R=500 \mathrm{kHz}$ memory cycling rate. We see from this figure that a throughput of 200 pairs/sec can be sustained out to an end-to-end path length $(2 L)$ of $50 \mathrm{~km}$, with a loss-limited fidelity of $97.5 \%$.

\section{DISCUSSION}

We have described a single-hop, long-distance, high-fidelity quantum communication system whose loss-limited operating range - without entanglement purification or quantum error correction - extends well beyond that of previous quantum repeater proposals. At $2 L=50 \mathrm{~km}$ there is $20 \mathrm{~dB}$ end-to-end loss in our system example, yet, because of the nondestructive memory-loading verification, the ultrabright nature of our entanglement source, and our ability to employ the low-loss wavelength window in standard telecommunication fiber, we can sustain appreciable throughputs and high fidelity.

Of course, this analysis has neglected any additional degradations that may arise from residual phase errors that are not common-moded out by our time-division multiplexing scheme. Likewise, the fidelity result we have reported applies to singlet-based teleportation assuming perfect Bell-state measurements, and perfect post-Bell-measurement 

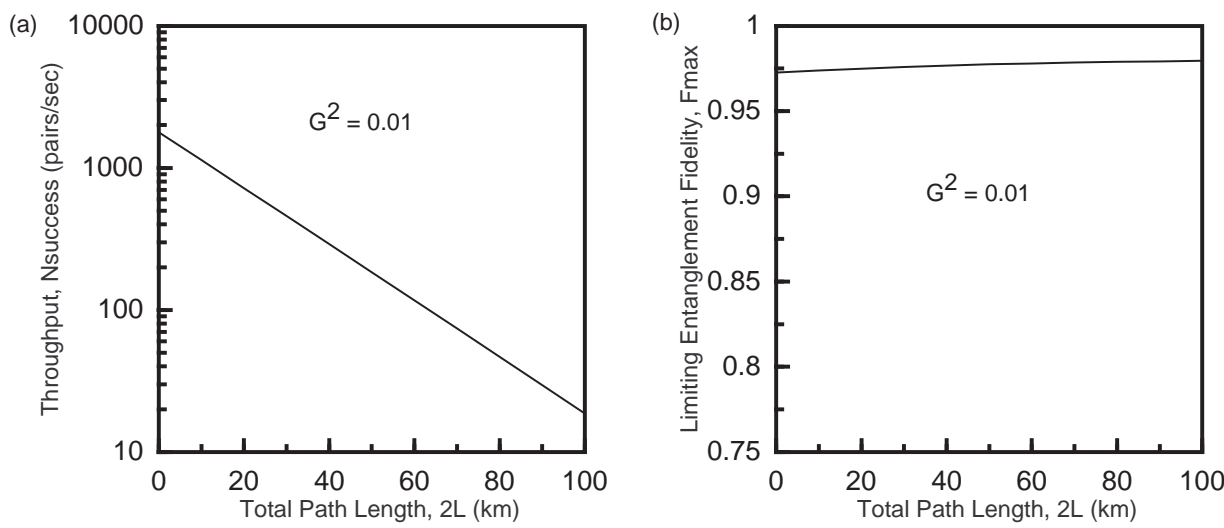

Figure 5. Figures of merit for Fig. 1 configuration. Left (a), throughput, $N_{\text {success }}$, vs. total path length, $2 L$. Right (b), limiting entanglement fidelity, $F_{\max }$, vs. total path length, $2 L$. All curves assume OPAs operating at $1 \%$ of their power thresholds, $5 \mathrm{~dB}$ of excess loss per $P$-to- $M$ block connection, and $0.2 \mathrm{~dB} / \mathrm{km}$ fiber-propagation loss.

state transformation. Imperfections in any of these areas will reduce the teleportation fidelity that can be achieved. Nevertheless, the Fig. 1 configuration offers substantial promise for bringing singlet-based teleportation from a conditional demonstration in the laboratory to a viable quantum communication system.

\section{ACKNOWLEDGEMENTS}

This research was supported in part by U.S. Army Research Office Grant DAAD1900-1-0177. The author acknowledges fruitful technical discussions with Phil Hemmer, Prem Kumar, Seth Lloyd, Selim Shahriar, Franco Wong, and Horace Yuen.

\section{REFERENCES}

1. J. H. Shapiro and N. C. Wong, An ultrabright narrowband source of polarization-entangled photon pairs, J. Opt. B: Quantum Semiclass. Opt. 2:L1 (2000).

2. S. Lloyd, M. S. Shahriar, and P. R. Hemmer, Teleportation and the quantum Internet, submitted to Phys. Rev. A (quant-phy/003147).

3. C. H. Bennett, G. Brassard, C. Crépeau, R. Josza, A. Peres, and W. K. Wootters, Teleporting an unknown quantum state via dual classical and Einstein-Podolsky-Rosen channels, Phys. Rev. Lett. 70:1895 (1993).

4. D. Bouwmeester, J.-W. Pan, K. Mattle, M. Eibl, H. Weinfurter, and A. Zeilinger, Experimental quantum teleportation, Nature 390:575 (1997).

5. D. Bouwmeester, K. Mattle, J.-W. Pan, H. Weinfurter, A. Zeilinger, and M. Zukowski, Experimental quantum teleportation of arbitrary quantum states, Appl. Phys. B 67:749 (1998).

6. P. G. Kwiat, E. Waks, A. G. White, I. Appelbaum, and P. H. Eberhard, Ultrabright source of polarization-entangled photons, Phys. Rev. A 60:R773 (1999).

7. P. Kumar, Quantum frequency conversion, Opt. Lett. 15:1476 (1990).

8. J. M. Huang and P. Kumar, Observation of quantum frequency conversion, Phys. Rev. Lett. 68:2153 (1992).

9. K. Bergman, C. R. Doerr, H. A. Haus, and M. Shirasaki, Sub-shot-noise measurement with fiber-squeezed optical pulses, Opt. Lett. 18:643 (1993).

10. J. I. Cirac, P. Zoller, H. J. Kimble, and H. Mabuchi, Quantum state transfer and entanglement distribution among distant nodes in a quantum network, Phys. Rev. Lett. 78:3221 (1997).

11. W. Dür, H.-J. Briegel, J. I. Cirac, and P. Zoller, Quantum repeaters based on entanglement purification, Phys. Rev. A 59:169 (1999).

12. N. C. Wong, K. W. Leong, and J. H. Shapiro, Quantum correlation and absorption spectroscopy in an optical parametric oscillator in the presence of pump noise, Opt. Lett. 15:891 (1990). 